\title{
Manejo quirúrgico de pacientes con Hidrosadenitis Supurativa. Experiencia del Hospital Clínico de la Universidad de Ghile
}

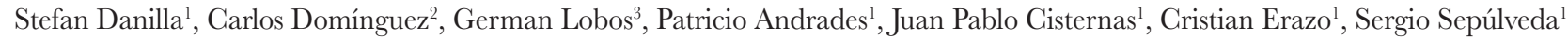

\section{RESUMEN}

Introducción: La hidrosadenitis supurativa es una enfermedad crónica de la piel en zonas que contienen glándulas apocrinas. Clásicamente se describe un manejo multimodal. La cirugía es una estrategia reconstructiva eficaz, frecuentemente postergada. No existen reportes sobre la experiencia a nivel nacional en el manejo de este grupo de pacientes.

Objetivos: Exponer los resultados de manejo quirúrgico reconstructivo de pacientes con diagnóstico de hidrosadenitis supurativa por parte de un equipo de cirugía plástica de un hospital clínico.

Material y Métodos: Serie de casos retrospectiva de pacientes con diagnóstico de hidrosadenitis supurativa con manejo quirúrgico. Se realizó una revisión del historial clínico de cada paciente y se elaborando un análisis descriptivo.

Resultados: Trece pacientes, 7 (53,8\%) de sexo femenino,

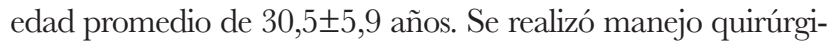
co de 17 lesiones, siendo las más frecuentes 9 (52,9\%)Axilar y $5(29,4 \%)$ Inguino-perianal. Se realizó drenaje-curaciones en 2 $(11,8 \%)$ y resección-cobertura en $15(88,2 \%), 14(82,3 \%)$ con colgajos y $2(11,8 \%)$ injertos.

Discusión: El manejo quirúrgico es una alternativa terapéutica eficaz para el control de la hidrosadenitis supurativa, presentando buenos resultados estéticos y funcionales con bajas complicaciones.

Palabras clave: Hidrosadenitis supurativa; cirugía plástica; tratamiento; cirugía reconstructiva, colgajos
${ }^{1}$ Cirujano plástico, Equipo de Cirugía Plástica y Reparadora. Departamento de Cirugía. Hospital Clínico de la Universidad de Chile, Santiago, Chile ${ }^{2}$ Fellow de Investigación en cirugía Plástica, Departamento de Cirugía. Hospital Clínico de la Universidad de Chile, Santiago, Chile

${ }^{3}$ Residente de Cirugía General, Universidad de Chile, Santiago, Chile

Correspondencia: Stefan Danilla Enei. Correo electrónico:

drstefandanilla@gmail.com, Teléfono: +56991580960 Dirección:

Santos Dumont 999, Independencia, Santiago, Chile. Código Postal:

8380456.

\section{SUMMARY}

Introduction: Hidradenitis suppurativa is a chronic skin diseases of areas that contain apocrine glands. Classically a multimodal management is described. Surgery is an effective treatment, often postponed.

Objectives: Present results of reconstructive surgical management of patients with hidradenitis suppurativa by a plastic surgery team of a clinical hospital.

Material and methods: Retrospective case series of patients with hidradenitis suppurativa with surgical management. A review of the clinical history of each patient was carried out and a descriptive analysis was carried out.

Results: 13 patients, $7(53.8 \%)$ female, mean age of $30.5 \pm$ 5.9 years. Surgical management of 17 lesions was performed, the most frequent lesions were $9(52.9 \%)$ Axillary and $5(29.4 \%)$ Inguino-perianal. Drainage were performed in $2(11.8 \%)$ and resection-coverage in $15(88.2 \%), 14(82.3 \%)$ with flaps and 2 $(11.8 \%)$ grafts.

Discussion: Surgical management is an effective therapeutic alternative for the management of hidradenitis suppurativa, presenting good esthetic and functional results with low complications.

Key words: Hidradenitis suppurativa plastic surgery, treatment, reconstructive surgery, flaps 
$\mathrm{L}$ a hidrosadenitis supurativa (HS) también conocida como acné inversa (enfermedad de Verneuil, apocrinitis), consiste en una enfermedad inflamatoria sistémica crónica, recurrente y debilitante ${ }^{1-5}$ que si bien se manifiesta en el folículo piloso, en un porcentaje relevante se asocia a otros procesos denominados comorbilidades, como obesidad, síndrome metabólico, espondiloartritis y enfermedad inflamatoria intestinal ${ }^{2-5}$. Se caracteriza por una adenitis apocrina ${ }^{6}$, por lo que afecta principalmente a territorios de piel que contienen glándulas sudoriparas apocrinas como axilas, región perineal, pliegue infra mamario y región púbica, ${ }^{2,3}$. Se presenta con mayor frecuencia en pacientes de sexo femenino, después de la pubertad, entre la $2^{\circ}$ y $3^{\circ}$ década de la vida con una incidencia del $1-4 \% 0^{2,3}$, con una fuerte repercusión en el ámbito psicológico de los pacientes, debido a las lesiones características de la enfermedad ${ }^{1,3}$.

$\mathrm{Su}$ etiopatogenia radica fundamentalmente en un desequilibrio del sistema inmunológico, de una persona predispuesta genéticamente, el cual se ve desencadenado o exacerbado por factores exógenos (obesidad, tabaco y hormonas), produciendo una respuesta inflamatoria del folículo pilo-sebáceo ${ }^{4,7}$.

Es una patología de diagnóstico clínico que a menudo, se confunde con carbunclo, quiste epidermoide, erisipela, linfogranuloma venéreo, granuloma inguinal, forúnculos o forunculosis ${ }^{2}$. Su diagnóstico se basa en tres características clínicas: lesiones típicas, localización típica, y recaída y cronicidad (2 eventos en 6 meses) de lesiones ${ }^{1,3}$. Dentro de las lesiones típicas encontramos doble comedones y nódulos subcutáneos dolorosos que pueden progresar a abscesos, fistulas con descarga de pus, fibrosis de la piel y cicatrices retractiles que limitan la movilidad de las extremidades. Son estas características clínicas las que permiten clasificar la enfermedad según los estadios clínicos de Hurley (Tabla 1) ${ }^{1}$.

Los objetivos del tratamiento de la HS incluyen la prevención de nuevas lesiones, manejo de lesiones recientes de forma temprana y eficaz, y la eliminación de los nódulos y tractos sinusales existentes ${ }^{2}$. El manejo global incorpora medidas generales como lo son cambio en el estilo de vida; eliminar el hábito tabáquico, pérdida de peso y alimentación saludable, medicamentos tanto tópicos como sistémicos; lociones tópicas, antibióticos, hormono terapia, retinoides, inmunosupresores y terapia biológica, y por último el manejo quirúrgico, que a pesar que no se considera curativa, puede extirpar la enfermedad con la consecuente mejoría anatómica, estética y psicológica para él o la paciente y con una baja tasa de recidiva que fluctúa entre $0-15 \%$ dependiendo de la estrategia terapéutica utilizada ${ }^{8-10}$. La decisión de la conducta terapéutica a implementar, dependerán de los estadios de Hurley ${ }^{2,11}$, que van desde medidas generales en los estadios más precoces hasta la cirugía, la cual está indicada básicamente en estadios avanzados de la enfermedad y estadios iniciales que no responden a tratamiento médico ${ }^{1}$. Previo a la realización de cualquier intervención quirúrgica, se recomienda lograr un período de mínima inflamación y actividad de la enfermedad, lo cual minimiza el trauma, mejora los resultados y disminuye la recurrencia ${ }^{3,5,7}$. Desafortunadamente el manejo quirúrgico, se emplea posterior al uso de numerosos ciclos de terapia farmacológica, retrasando su indicación ${ }^{3}$.

Dentro de las diferentes opciones quirúrgicas se encuentras la incisión y drenaje, destechamiento, marsupialización, exéresis localizada y exéresis amplia, junto con diferentes tipos de cierre de la región afectada, como son el cierre primario, secundario, colgajo e injerto. El tipo de cirugía y cierre será seleccionado en función de la zona implicada y del grado de afectación ${ }^{1,11}$.

A nivel internacional existen diversas series clínicas de manejo quirúrgico de pacientes con HS utilizando de diferentes alternativas reconstructivas, a nivel nacional Herane $e t a^{1}$, presento una revisiones del tema y existen escasos reportes de pacientes sometidos a manejo quirúrgico, destacando una serie de pacientes pediátricos presentada por Silva-Feistner el 201612 y el reporte de Dagnino et $a l^{13}$ describiendo el uso de colgajos perforantes en el manejo de HS axilar y esternal. 
Nuestro enfoque como equipo constituye en la resección amplia de las lesiones y reconstrucción, de tal forma de realizar cirugía con intención curativa.

El objetivo del estudio es presentar una serie clínica de pacientes con diagnóstico de Hidrosadenitis Supurativa, manejados en forma quirúrgica por un equipo de cirugía plástica de un Hospital Clínico universitario.

\section{Materiales y Métodos}

Serie de casos retrospectiva de pacientes con diagnóstico de hidrosadenitis supurativa con manejo quirúrgico, obtenida del registro de pacientes quirúrgicos del equipo de cirugía plástica entre los años 2011 y 2017. Se incluyó a todos los pacientes con diagnostico confirmado de HS, sometidos a manejo quirúrgico y que fueron controlados por el equipo de cirugía plástica de nuestro centro. Se excluyó a pacientes con diagnósticos dudoso e historia clínica incompleta.

Se revisó el historial clínico de cada paciente, recopilando variables demográficas, antecedentes clínicos y de tratamiento. Elaborando un análisis descriptivo, utilizando medidas de tendencia central, dispersión y asociación para evaluar la distribución entre grupos. Para lo cual se utilizó STATA 13, considerando p <0,05 estadísticamente significativo.

Se considero como procedimientos quirúrgicos el drenaje de lesiones abscedadas y la resección completa de piel y glándulas apocrinas de las regiones comprometidas. Las estrategias reconstructivas utilizadas consideraron el cierre por segunda intención en el caso de lesiones drenadas y la cobertura de los defectos cutáneos resecados utilizando colgajos e injertos.

\section{Resultados}

Se incluyeron 13 pacientes, 7 (53,84\%) de sexo femenino, edad promedio de 30,5 $\pm 6,3$ años [22-41], IMC $27,7 \pm 3,3 \mathrm{Kg} / \mathrm{m}^{2}$ y $3,8 \pm 2,2$ años de evolución de la enfermedad. Observándose un menor tiempo de evolución de la enfermedad en pacientes de sexo femenino (Mujeres $2,75 \pm 0,87$ v/s 4,67 $\pm 2,60$ en hombres, $p<0,033$. No se observó diferencias de edad ni IMC por sexo $(\mathrm{p}=0,986$ y $\mathrm{p}=0,569$ respectivamente).
Tabla 1

Estadios de Hurley para Hidrosadenitis supurativa.

\begin{tabular}{lll}
\hline Estadio & Abscesos & Trayectos fistulosos \\
\hline I & Uno o más & No \\
II & Separados y recurrentes & Escasos \\
III & Múltiples & Múltiples \\
\hline
\end{tabular}

Nueve $(69,23 \%)$ pacientes presentaron compromiso en múltiples regiones corporales, siendo las ubicaciones más frecuentes: inguinal en 8 (61,54\%), 7 (53,85\%) Axilar y $5(38,46 \%)$ Genital, no observándose diferencias en la distribución según sexo $(\mathrm{p}=0,897)$ (Tabla 2).

Del total de pacientes intervenidos $12(92,31 \%)$ recibieron manejo medico previo al quirúrgico, de los cuales 11 $(84,62 \%)$ recibió tratamiento tópico, $11(84,62 \%)$ tratamiento oral (antibióticos y retinoides) y $3(23,08 \%)$ tratamiento biológico (Infliximab). Nueve $(62,23 \%$ ) pacientes presentaban además el antecedente de haber sido sometidos a manejo quirúrgico mediante drenaje y cierre por segunda intención en al menos en una oportunidad.

En total se realizó manejo quirúrgico de 17 lesiones en regiones corporales diferentes, 9 (76,92\%) pacientes requirieron manejo quirúrgico de una sola lesión. Las regiones corporales intervenidas más frecuentes fueron $9(52,94 \%)$ Axilares, 5 (29,41\%) Inguino-perianal, $2(11,76 \%)$ abdominal y esternal 1 (5,88\%) (Grafico 1$)$. Las cuales presentaban compromiso clínico compatible con lesiones tipo III de Hurley en $13(76,47 \%)$ y tipo II en $4(23,52 \%)$ casos, presentando al momento de la intervención un estado inflamatorio activo en $10(58,52 \%)$ casos y $10(58,52 \%)$ habían sido drenadas previamente en episodios anteriores.

Las lesiones que presentaban un estado clínico activo al ingreso, recibieron tratamiento antibiótico previo a la intervención quirúrgica y en $5(50 \%)$ casos se realizó drenaje quirúrgico previo a la intervención definitiva.

Las lesiones tratadas presentaron un área resecada promedio de $150,7 \pm 133,2 \mathrm{~cm}^{2}$. Se realizó resección de la lesión con cobertura del defecto en $15(88,23 \%)$ y drenaje - cierre por segunda intención en $2(11,76 \%)$ casos. Las estrategias reconstructivas utilizadas en los casos resecados fueron: $13(86,67 \%)$ colgajos, 1 (6,67\%) injerto y 1 $(6,67 \%)$ colgajos combinado con injerto. De los colgajos utilizados $6(42,86 \%)$ correspondieron a colgajos locales, 
Tabla 2

Tabla resumen de pacientes

\begin{tabular}{|c|c|c|c|c|c|c|c|c|c|}
\hline $\mathbf{N}^{\mathbf{o}}$ & Paciente & Sexo & Edad & IMC & Hurley & Zona & $\begin{array}{l}\text { Manejo } \\
\text { tratada }\end{array}$ & $\begin{array}{l}\text { Complicación } \\
\text { defecto }\end{array}$ & Seguimiento \\
\hline 1 & 1 & $\mathrm{~F}$ & 22 & 28 & III & $\begin{array}{l}\text { Axilar } \\
\text { izquierda }\end{array}$ & $\begin{array}{l}\text { Colgajo músculo } \\
\text { dorsal ancho } \\
\text { con isla cutánea }\end{array}$ & & 9 \\
\hline 2 & & & 23 & 27 & III & $\begin{array}{l}\text { Axilar } \\
\text { derecha }\end{array}$ & $\begin{array}{l}\text { Colgajo músculo } \\
\text { dorsal ancho } \\
\text { con isla cutánea }\end{array}$ & & 7 \\
\hline 3 & 2 & M & 22 & 27 & III & Esternal & $\begin{array}{l}\text { Colgajo libre } \\
\text { antero lateral } \\
\text { del muslo }\end{array}$ & & 76 \\
\hline 4 & & & 28 & 23,5 & III & Abdominal & Colgajo local & Sangrado & 10 \\
\hline 5 & & & 28 & 23,5 & III & $\begin{array}{l}\text { Inguinal - } \\
\text { perineal }\end{array}$ & $\begin{array}{l}\text { Colgajo músculo } \\
\text { recto abdominal } \\
\text { con isla cutánea } \\
\text { vertical + IDE } \\
\text { + Colgajo Local }\end{array}$ & $\begin{array}{l}\text { Dehiscencia } \\
\text { herida }\end{array}$ & 9 \\
\hline 6 & 3 & $\mathrm{~F}$ & 26 & 24,4 & II & $\begin{array}{l}\text { Axilar } \\
\text { izquierda }\end{array}$ & $\begin{array}{l}\text { Colgajo perforante } \\
\text { de arteria toraco } \\
\text { dorsal }\end{array}$ & Cicatriz retráctil & 17 \\
\hline 7 & 4 & M & 26 & 28,7 & III & $\begin{array}{l}\text { Axilar i } \\
\text { izquierda }\end{array}$ & $\begin{array}{l}\text { Colgajo perforante } \\
\text { de arteria toraco } \\
\text { dorsal }\end{array}$ & $\begin{array}{l}\text { Sufrimiento } \\
\text { parcial }\end{array}$ & 44 \\
\hline 8 & & & 28 & 28 & III & Axilar & $\begin{array}{l}\text { Colgajo Local } \\
\text { derecha }\end{array}$ & & 28 \\
\hline 9 & 5 & $\mathrm{~F}$ & 32 & 32 & II & Inguinal & Colgajo Local & & 37 \\
\hline 10 & 6 & M & 37 & 37 & III & $\begin{array}{l}\text { Axilar } \\
\text { derecha }\end{array}$ & Colgajo Local & $\begin{array}{l}\text { Dehiscencia } \\
\text { herida }\end{array}$ & 19 \\
\hline 11 & 7 & M & 35 & 25 & II & $\begin{array}{l}\text { Perine - } \\
\text { gluteo }\end{array}$ & IDE & $\begin{array}{l}\text { Pérdida parcial } \\
\text { y Residiva }\end{array}$ & 28 \\
\hline 12 & 8 & $\mathrm{~F}$ & 33 & 27 & III & $\begin{array}{l}\text { Axilar } \\
\text { derecha }\end{array}$ & $\begin{array}{l}\text { Colgajo perforante } \\
\text { de arteria toraco } \\
\text { dorsal }\end{array}$ & & 20 \\
\hline 13 & 9 & $\mathrm{~F}$ & 40 & 27,7 & II & $\begin{array}{l}\text { Axilar i } \\
\text { zquierda }\end{array}$ & (Drenaje) & & 6 \\
\hline 14 & 10 & M & 30 & 25,6 & III & Abdominal & Colgajo Local & & 5 \\
\hline 15 & 11 & M & 41 & 27,6 & III & $\begin{array}{l}\text { Inguinal - } \\
\text { perineal }\end{array}$ & (Drenaje) & & 7 \\
\hline 16 & 12 & $\mathrm{~F}$ & 40 & 29 & III & $\begin{array}{l}\text { Axilar } \\
\text { derecha }\end{array}$ & $\begin{array}{l}\text { Colgajo musculo } \\
\text { dorsal ancho con } \\
\text { isla cutánea }\end{array}$ & & 5 \\
\hline 17 & 13 & $\mathrm{~F}$ & 28 & 31,2 & III & Inguinal & Colgajo Local & & 8 \\
\hline
\end{tabular}


Tabla 3

Manejo quirúrgico de regiones corporales con hidrosadenitis supurativa

\begin{tabular}{ll}
\hline Manejo realizado & N (\%) \\
\hline Drenaje y curación & $2(11,76 \%)$ \\
\hline Resección y cobertura & $15(88,24 \%)$ \\
\hline Injerto & $2(11,76 \%)$ \\
\hline Colgajo local & $5(29,41 \%)$ \\
\hline Colgajo musculo-cutáneo & $4(23,53 \%)$ \\
Musculo dorsal ancho con isla cutánea & $3(17,65 \%)$ \\
Musculo recto abdominal con isla cutánea vertical & $1(5,83 \%)$ \\
\hline Perforantes & $3(17,65 \%)$ \\
\hline Colgajo perforante de arteria toraco dorsal & \\
\hline Colgajo Libres & $2(11,76 \%)$ \\
Antero lateral de muslo &
\end{tabular}

VRAM: Colgajo musculo cutáneo de recto abdominal con isla cutánea vertical; TDAP: Colgajo perforante de arteria toraco dorsal; ALT: Colgajo Libre antero lateral de muslo

$4(28,57 \%)$ colgajos musculo-cutáneos, 3 (21,42\%) colgajos perforantes y $2(14,29 \%)$ colgajos libres (Figura 1) (Tabla 3).

La serie registra $6(35,29 \%)$ pacientes con complicaciones leves, $2(11,76 \%)$ dehiscencias parciales de herida operatoria, $1(5,88 \%)$ sangrado, $1(5,88 \%)$ sufrimiento parcial de colgajo y $1(5,88 \%)$ de injerto. Y un paciente curso con una cicatriz axilar retráctil, que fue manejada con kinesioterapia.

Cinco $(29,41 \%)$ pacientes fueron sometidos a revisión quirúrgica, 2 (11,76\%) avances de colgajos, 2 (11,76\%) plastias de cicatrices consideradas como poco estéticas y $1(5,88 \%)$ re-injerto. No se registró complicaciones mayores ni mortalidad

La serie presento un seguimiento promedio de 16,7 $\pm 19,6$ meses, observándose un caso de recidiva local, en un paciente con antecedente de VIH.

\section{Discusión}

La hidrosadenitis supurativa es una patología inflamatoria crónica y recurrente de la piel que, junto con presentar lesiones clínicamente características, se asocia a considerables efectos psicológicos para los pacientes. El manejo quirúrgico se suele reservar para estadios avanzado de la enfermedad.

\section{Gráfico 1}

Distribución de regiones anatómicas comprometidas con Hidrosadenitis Supurativa
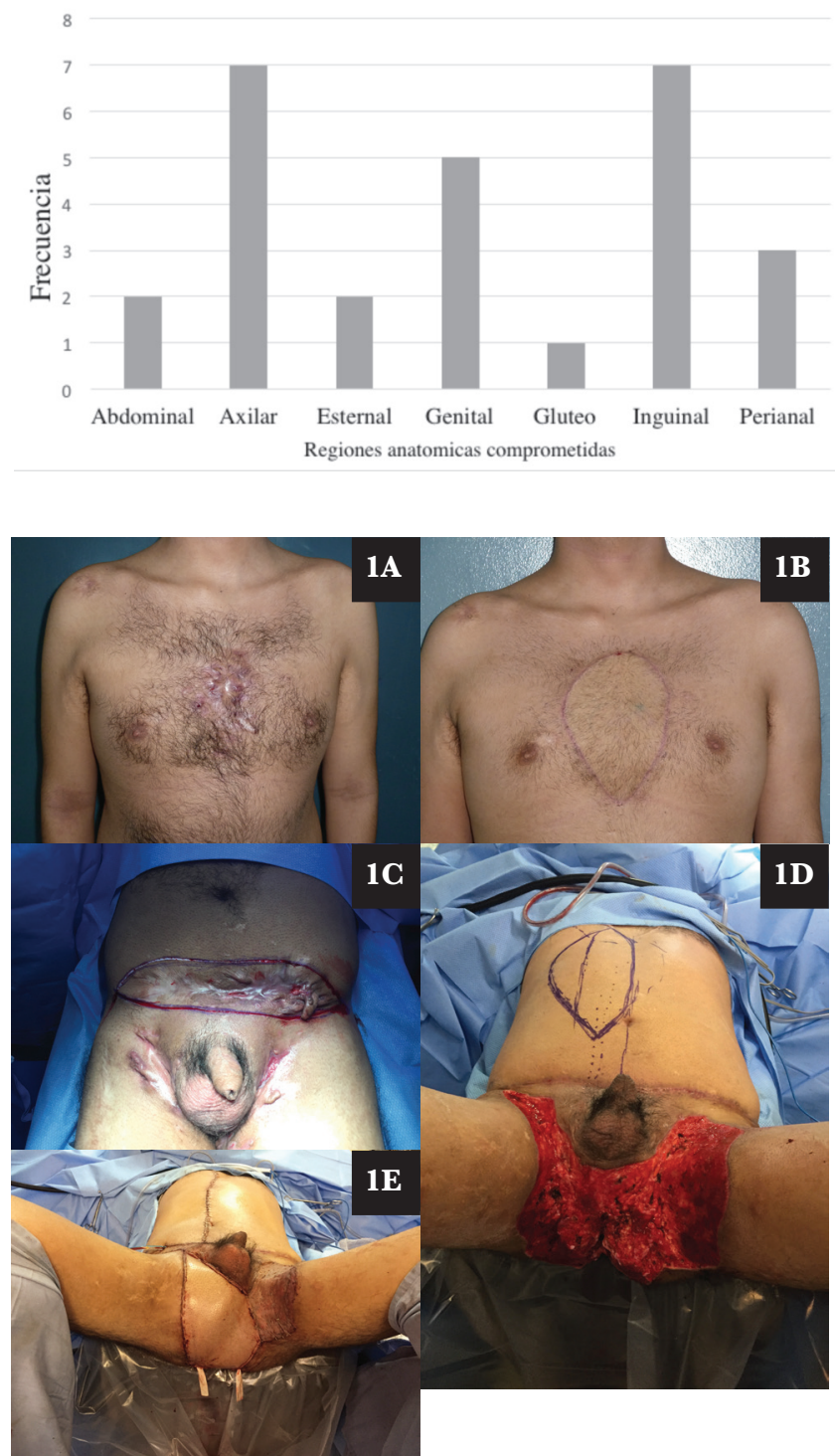

\section{Figura 1}

A. Paciente de 22 años sexo masculino con antecedente de sobrepeso, que presenta cuadro de Hidrosadenitis supurativa en región esternal (Hurley III) inguinal y abdominal (Hurley I).

B. Se realiza resección extensa de piel y tejido celular subcutáneo y posterior cobertura con colgajo libre ALT.

C. Seis años después es reevaluado debido a progresión de lesiones en región abdominal e inguino-perineal activas.

D. Se realiza resección de región abdominal horizontal y cobertura con avance de colgajo tipo abdominoplastía; durante la misma hospitalización se ejecuta extensa resección de piel inguinal bilateral y perianal.

E. Realizando cobertura cutánea mediante el uso combinado de colgajos musculo-cutaneo (VRAM), colgajos locales e IDE. 
$\mathrm{Al}$ igual que lo reportado por otras series ${ }^{14-16}$ de pacientes sometidos a manejo quirúrgico de HS, se observa un predominio de sexo femenino, con una edad que fluctúa entre los 20-40 años, una tendencia al sobrepeso y obesidad (92,3\%), siendo las regiones inguinales $(61,54 \%)$ y axilares $(53,85 \%)$ las con mayor presencia de lesiones compatibles con HS.

Los pacientes que llegan a manejo quirúrgico suelen haber recibido tratamiento médico por un tiempo prolongado y haber sido sometidos a drenajes quirúrgicos previos. A diferencia de lo reportado por Kagan et al ${ }^{14}$ el tiempo de evolución promedio de cuadro hasta la resección y cobertura en nuestra experiencia es menor a lo reportado (3,8 v/s 6,7 años), observándose en ambas series una mayor postergación de resolución quirúrgica en pacientes de sexo masculino, siendo esta diferencia estadísticamente significativa en ambas series $(\mathrm{p}=0,033$ y $\mathrm{p}=0,001$ respectivamente).

Nuestra experiencia el manejo quirúrgico se realizó principalmente en lesiones avanzadas Hurley III (76,47\%), con compromiso Axilar e inguinal, siendo la resección y posterior cobertura con colgajos la estrategia más utilizada (88,24\%). Alhabri el 201216, describe un $49 \%$ de lesiones axilares, $36 \%$ Inguinales, $16 \%$ Perineales y $2 \%$ en tronco y glúteo respectivamente, siendo estas resecadas y los defectos cutáneos reparados mediante el uso de colgajos fasciocutaneos, muscuocutaneos e Injertos.

Las estrategias reconstructivas utilizadas se basaron en lograr una cobertura cutánea adecuada, tomando en consideración resultados estéticos y funcionales. En la región axilar se utilizó principalmente el colgajo perforante de la arteria Toracodorsal (TDAP) $(33,33 \%)$ y el colgajo musculo cutáneo de Dorsal Ancho $(33,33 \%)$ (Figura 2), se realizó un $(11,11 \%)$ colgajo libre antero lateral de muslo (ALT). En la literatura existen diversas series que respaldan la reconstrucción con colgajos, Laredo et al15, el 2010 publica su experiencia reconstructiva en pacientes colgajo TDAP, destacando ausencia de recurrencia, con buenos resultados funcionales y estéticos y baja morbilidad del sitio donante, Alhabri et $a l^{16}$ describe el uso de colgajos fascio-cutaneos de rotación $(30,4 \%)$ y transposición $(39,1 \%)$ como las principales estrategias reconstructivas, describiendo además el uso de un colgajo libre ALT. Ching $e t ~ a l^{17}$ describe además el uso de colgajos

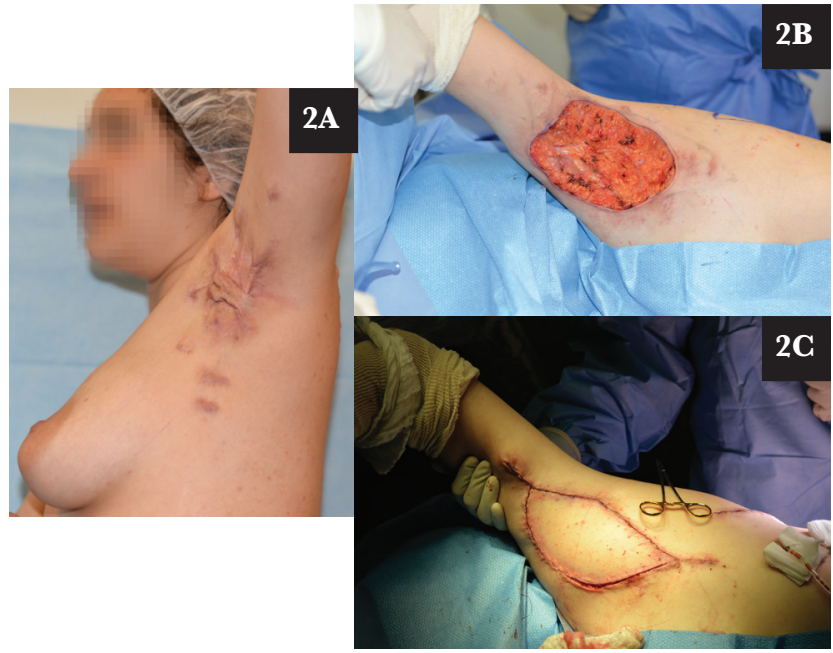

\section{Figura 2}

A. Paciente de sexo femenino con antecedentes de sobrepeso y tratamiento prolongado tópico y sistémico por Hidrosadenitis supurativa en ambas regiones axilares, con mayor compromiso a izquierda (Hurley III).

B. Se realiza resección de tejido comprometido, sin profundizarse en fosa axilar, para no dañar estructuras vasculo nerviosas.

C. Cobertura de defecto con colgajo musculo-cutáneo de dorsal ancho.

de transposición desde el brazo, reportando buenos resultados, estéticos y funcionales, con baja morbilidad en zona dadora.

En la región inguino-perineal se describe el cierre primario como principal estrategia reconstructiva, seguido por el uso injertos de piel parcial (IDE) y de colgajos fascio-cutaneos de rotación ${ }^{9,16}$. En nuestra experiencia el uso de colgajos fascio-cutaneos fue el principal método usado y en un caso, dado el compromiso inguino-perineal bilateral, se realizó la cobertura del defecto resecado utilizando en forma combinada un colgajo de musculo recto abdominal con paleta cutánea vertical (VRAM), asociado a colgajos locales de avance de muslos e injerto de piel parcial.

En la región abdominal el uso de colgajos de avance tipo abdominoplastía es la estrategia más utilizada en nuestra experiencia y por otros autores ${ }^{16,18}$.

El desarrollo de complicaciones quirúrgicas post operatorias es un riesgo propio de esta estrategia terapéutica, al igual que lo presentado por Jasen et al 201618 en nuestra experiencia no se observó complicaciones mayores asociadas a la intervención quirúrgica. Si 


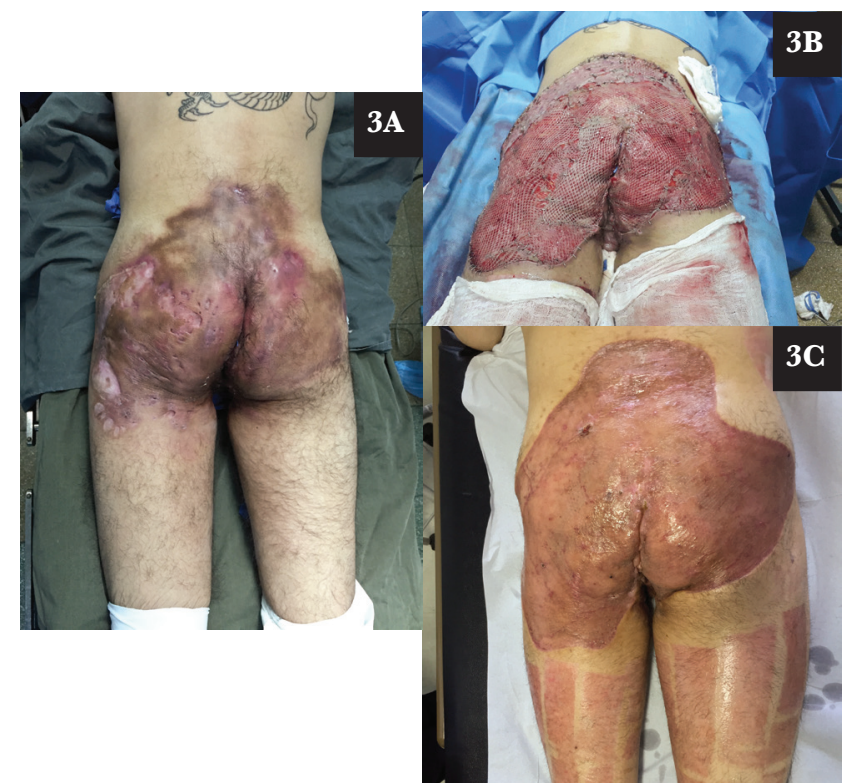

Figura 3

A. Paciente sexo masculino de 35 años, antecedente de $\mathrm{VIH}(+)$, con compromiso extenso de Hidrosadenitis supurativa de región perineal y glútea.

B. Se realiza resección del tejido comprometido e injerto de piel parcial.

C. Control ambulatorio al alta.

bien $5(29,4 \%)$ casos presentaron alguna complicación menor local durante los primeros 30 días de post operatorio, siendo la dehiscencia de herida operatoria y el sufrimiento parcial del colgajo las complicaciones más frecuentes. Durante el post operatorio tardío se observó el desarrollo de dos complicaciones una de las cuales correspondió a una secuela de cicatriz (cicatriz retráctil) manejada con quinesioterapia y un paciente presento recidiva local a nivel glúteo. No se observó el desarrollo de otras complicaciones descritas con alta frecuencia, tales como la infección de herida operatoria $(10,9 \%)$ ni el dolor post operatorio excesivo $(32,7 \%)^{18}$.

Existe concordancia entre diversas series respecto a que el manejo quirúrgico basado en drenaje y cierre por segunda intención presenta mayor tasa de recidiva $(27 \%)$, seguido por la resección y cierre primario $(22 \%)^{9,10}$, esto atribuido a que se asocia a resecciones más limitadas. Respecto al uso de colgajos existen reportes de series con tasas de recidiva variable $0-39 \%{ }^{15,16}$. En nuestra experiencia se observó un caso de recidiva $(5,88 \%)$, en un paciente inmunosuprimi- do con extenso compromiso perineal y glúteo, el cual había sido manejado con IDE (Figura 3)

Esta baja recidiva observada se podría atribuir a una resección completa del tejido clínicamente comprometido, hasta asegurar márgenes sanos de resección, lo cual es concordante con lo descrito por Kegan ${ }^{14}$, otros autores describen resecciones radicales de todo el tejido piloso hasta el plano aponeurótico ${ }^{14,15,19}$, las cuales, si bien presentan bajas tasas de recidiva, generan defectos de mayor tamaño, más complejos de cubrir.

\section{Conclusión}

El manejo quirúrgico es una estrategia terapéutica eficaz para el manejo de la hidrosadenitis supurativa, que ofrece buenos resultados estéticos y funcionales, con bajas complicaciones. Por tal motivo creemos que es una alternativa que debe ser considerada en la forma precoz en pacientes con compromiso avanzado y refractario al tratamiento médico, evitando de este modo la progresión de la extensión de las lesiones, dificultando su manejo. 


\section{REFERENCIAS}

1. Herane MI, Alarcon R. Hidrosadenitis supurativa y procesos afines. Mas dermatologia. 2012 Jan 2;16(16):4-12

2. Yue E, Alhusayen R, Langsang P, Shear N, Yeung J. What is hidradenitis suppurativa? Canadian Family Physician. 2017;63:11420

3. Med RKAB, DMSci GBEJM. Treatments for Hidradenitis Suppurativa. Clinics in Dermatology. Elsevier B.V; 2016;35(2):21824

4. Martorell A, García-Martínez FJ, Jiménez-Gallo D, Pascual JC, Pereyra-Rodriguez J, Salgado L, et al. Actualización en hidradenitis supurativa (I): epidemiología, aspectos clínicos y definición de severidad de la enfermedad. Actas dermosifiliogríaficas. AEDV; 2015 Nov 1;106(9):703-15

5. Martorell A, Caballero A, Lama YG, Jiménez-Gallo D, Serrano ML, Miranda J, et al. Manejo del paciente con hidradenitis supurativa. Actas dermosifiliogríaficas. Elsevier; 2016 Sep 1;107(Supplement 2):32-42

6. Sugio Y, Tomita K, Hosokawa K. Reconstruction after Excision of Hidradenitis Suppurativa. Plastic and Reconstructive Surgery Global Open. 2016 Nov;4(11):e1128

7. Martorell A, Garc a FJ, Jim nez-Gallo D, Pascual JC, PereyraRodriguez J, Salgado L, et al. Actualización en hidradenitis supurativa (ii): aspectos terap?uticos. Actas Dermo-Sifiliogr?ficas. 2015 Nov; 106(9):716-24

8. Unal G, Yirmibesoglu O, Ozdemir J, Hasdemir M. Superior and inferior gluteal artery perforator flaps in reconstruction of gluteal and perianal/ perineal hidradenitis suppurativa lesions. Microsurgery. 2011 Aug 23;31(7):539-44

9. Balik E, Eren T, Bulut T, Büyükuncu Y, Bugra D, Yamaner S. Surgical Approach to Extensive Hidradenitis Suppurativa in the Perineal/ Perianal and Gluteal Regions. World J Surg. 2008 Dec 6;33(3):481-7
10. Mehdizadeh A, Hazen PG, Bechara FG, Zwingerman N, Moazenzadeh M, Bashash M, et al. Recurrence of hidradenitis suppurativa after surgical management: A systematic review and meta-analysis. YMJD. Elsevier; 2015 Nov 1;73(Supplement 1):S70-7

11. PedrazJ, Dauden E. Manejo práctico de la hidrosadenitis supurativa. Actas dermosifiliogríaficas. 2008;99:101-10

12. Silva-Feistner M, Salomone C, Silva S, Vial-Letelier VN. Hidradenitis supurativa prepuberal: presentaci?n de 2 casos cl?nicos. Revista Chilena de Pediatr?a. 2016 May;87(3):193-8

13. Dagnino BL, Salisbury MC, Cifuentes IJ, Rodriguez JR, Searle SV. Uso de colgajos perforantes para el tratamiento de hidradenitis supurativa axilar y esternal graves. Revista Chilena de Cirugía

14. Kagan RJ, Yakuboff KP, Warner P, Warden GD. Surgical treatment of hidradenitis suppurativa: A 10-year experience. Surgery. 2005 Oct;138(4):734-41

15. Laredo Ortiz C, López Castillo V, Solesio Pilarte F, Lorda Barraguer E. Experience Using the Thoracodorsal Artery Perforator Flap in Axillary Hidradentitis Suppurativa Cases. Aesthetic Plast Surg. 2010 Jun 30;34(6):785-92

16. Alharbi Z, Kauczok J, Pallua N. A review of wide surgical excision of hidradenitis suppurativa. BMC Dermatology. 2012;12(1):9-17.

17. Ching DL, Mughal M, Papas A, Soldin M. Axillary Reconstruction for Hidradenitis Suppurativa with an Inner-Arm Transposition Flap Creating a Brachioplasty Effect. Arch Plast Surg. 2017;44(3):228-33.

18. Janse I, Bieniek A, Horváth B, Matusiak Ł. Surgical Procedures in Hidradenitis Suppurativa. Dermatologic Clinics. Elsevier Inc; 2016;34:97-109.

19. Parks RW, Parks TG. Pathogenesis, clinical features and management of hidradenitis suppurativa. annals. 1997;79:83-9. 